\title{
Molecular mechanisms of the inhibitory effects of jiangu granule-containing serum on RANKL-induced osteoclastogenesis
}

\author{
YUNMEI HUANG ${ }^{1}$, YU LIN ${ }^{2}$, YINSHENG WU ${ }^{1}$, JIANWEI ZENG ${ }^{1}$, MEIYA HUANG $^{3}$, \\ SHIMING GUO ${ }^{1}$, WENJUAN LUO ${ }^{1}$, HAIMING LIN ${ }^{1}$ and YANPING LIN ${ }^{1}$ \\ ${ }^{1}$ Academy of Integrative Medicine, Fujian University of Traditional Chinese Medicine, Minhou Shangjie, Fuzhou, \\ Fujian 350122; ${ }^{2}$ Joint Surgery, Fuzhou No. 2 Hospital Affiliated Xiamen University, Fuzhou, Fujian 350007; \\ ${ }^{3}$ Fujian Provincial Key Laboratory of Integrative Medicine on Geriatrics, Fujian University of \\ Traditional Chinese Medicine, Minhou Shangjie, Fuzhou, Fujian 350122, P.R. China
}

Received December 21, 2016; Accepted July 26, 2017

DOI: $10.3892 / \mathrm{mmr} .2017 .7645$

\begin{abstract}
Postmenopausal osteoporosis (PMOP) is characterized by increased bone loss due to enhanced osteoclastogenesis and bone resorption. A Chinese herbal formula, jiangugranule (JG), exhibited great efficacy in the clinical treatment of PMOP. However, the molecular mechanisms underlying the therapeutic effects remain unclear. The present study aimed to examine the effects of JG-containing serum on receptor activator of nuclear factor- $\kappa \mathrm{B}(\mathrm{NF}-\kappa \mathrm{B})$ ligand (RANKL)-induced osteoclastogenesis. Osteoclast precursor RAW264.7 cells were cultured and treated with JG-containing serum in the presence of RANKL. Following 6 days of culture, the cells were stained with tartrate-resistant acid phosphatase and the rate of differentiation was calculated. In addition, cells were treated with JG-containing serum for 24, 48 and $96 \mathrm{~h}$ and total RNA and proteins were extracted for reverse transcription-quantitative polymerase chain reaction and western blot analysis to detect mRNA and protein expression, respectively, of key molecules in the RANK/RANKL signaling pathway, including RANK, tumor necrosis factor receptor-associated factor $6, \mathrm{NF}-\kappa \mathrm{B}$ (p50 and p52 subunits), c-Fos and nuclear factor of activated T cells, cytoplasmic 1 (NFATc1). The results revealed that JG-containing serum inhibited RANKL-induced osteoclastogenesis and reduced mRNA and protein expression of RANK, c-Fos and NFATc1. The results suggested that JG may regulate osteoclast differentiation through the RANK/RANKL signaling pathway, which may be a possible mechanism for the therapeutic effects of JG on PMOP.
\end{abstract}

Correspondence to: Professor Yanping Lin, Academy of Integrative Medicine, Fujian University of Traditional Chinese Medicine, 1 Qiuyang Road, Minhou Shangjie, Fuzhou, Fujian 350122, P.R. China

E-mail: linyanping1966@163.com

Key words: postmenopausal osteoporosis, jiangu granule, osteoclast, receptor activator of nuclear factor- $\mathrm{\kappa B}$ ligand

\section{Introduction}

Primary osteoporosis is a systemic skeletal disease that is characterized by low bone mass, microstructural damage of bone tissue and weakened bone strength (1). It may lead to a significant increase in fracture risk and may be a serious threat to the health of patients. Postmenopausal osteoporosis (PMOP) is one of the most common types of primary osteoporosis. Owing to the decrease of estrogen levels, osteoclastogenesis is enhanced, which results in dramatic bone loss and increased bone destruction (2-4). Pharmacological interventions for osteoporosis include antiresorptive agents that prevent bone resorption and anabolic agents that aid in new bone formation. Despite the multiple management options, there remain concerns for potential adverse effects of these agents (5). Discovery of the receptor activator of nuclear factor $-\kappa B$ (RANK)/RANK ligand (RANKL) signaling pathway, which is a key regulator of osteoclastogenesis and bone resorption, has led to a novel therapy that targets this pathway in the treatment of osteoporosis $(6,7)$.

In Traditional Chinese Medicine (TCM) it is believed that kidney deficiency is the main pathogenesis of PMOP, and spleen deficiency is also involved $(8,9)$. A TCM formula, jiangugranule (JG; which includes calcined Os Canitis, Epimediumbrevicornum Maxim and Davalliamariesii Moore ex Bak), exhibited great efficacy in the clinical treatment on PMOP by tonifying the kidney and invigorating the spleen. The authors previous studies have demonstrated that JG significantly improved both bone quantity and bone quality of PMOP model rats by reducing bone calcium loss and collagen degradation $(10,11)$. Owing to the direct relationship between bone decomposition and osteoclast activity, the present study focused on osteoclast differentiation and the key regulators. The effects of JG-containing serum on RANKL-induced osteoclastogenesis and the expression of key molecules in the RANK/RANKL signaling pathway were examined, including RANK, tumor necrosis factor receptor-associated factor 6 (TRAF6), nuclear factor- $\kappa \mathrm{B}$ (NF-kB), c-Fos and nuclear factor of activated T cells, cytoplasmic 1 (NFATc1), to investigate the mechanism of JG on preventing and treating PMOP. 


\section{Materials and methods}

Preparation of JG. JG is a TCM prescription that comprises calcined Os Canitis (500 mg), E. brevicornum Maxim (12 g), D. mariesii Moore ex Bak. (12 g), Cornusofficinalis Sieb.et Zucc. (9 g), Lyciumchinense Mill. (9 g), Dioscoreaopposita (9 g), Codonopsispilosula (10 g), Dipsacusasperoides C.Y. Cheng et T.M. Ai (10 g), Crocus sativus L. (2 g), Pericarpium Citri Reticulatae (6 g), Curcuma longa L. (10 g) and Carapax Testudinis (400 mg). The medicinal materials were purchased from Fujian Pharmaceutical Company (Fuzhou, China). All ingredients are made into traditional Chinese medicine granules by Fujian Academy of Traditional Chinese Medicine (Fujian, China). All the medicinal materials were broken into coarse powder and mixed together. The mixture was immersed in distilled water for $1 \mathrm{~h}$ and then boiled in a distillation apparatus for $2 \mathrm{~h}$. The extracting solution was concentrated to extract under a vacuum in a $50^{\circ} \mathrm{C}$ water bath, then cooled and stored at $4^{\circ} \mathrm{C}$ until use. A total of 1 gram of JG was equivalent to $2.99 \mathrm{~g}$ of crude medicines.

Analysis of JG by high-performance liquid chromatography (HPLC). An Agilent 1260 Liquid Chromatography system (Agilent Technologies, Inc., Santa Clara, CA, USA), equipped with a G1311C quaternary solvent delivery system, a G7617B autosampler and a G1315D diode array detector was used to detect icariin from E. brevicornum Maxim, morroniside and loganin from C.officinalis Sieb. et Zucc., andaurantiamarin from Pericarpium Citri Reticulatae. An Agilent HC-C18 $(25 \times 4.6 \mathrm{~mm} ; 5 \mu \mathrm{m})$ column connected with a Zorbax Extend guard column $(20 \times 4.6 \mathrm{~mm} ; 5 \mu \mathrm{m})$ was used. The column temperature was set at $30^{\circ} \mathrm{C}$. The mobile phase consisted of (A) acetonitrile and (B) water (v/v) using a linear gradient elution of $10-20 \% \mathrm{~A}$ at $0-15 \mathrm{~min}, 20-30 \% \mathrm{~A}$ at $15-20 \mathrm{~min}$ and $30 \% \mathrm{~A}$ at $20-35 \mathrm{~min}$. The flow rate was $1.0 \mathrm{ml} / \mathrm{min}$, and $10 \mu \mathrm{l}$ of sample was injected. Ultraviolet detection wavelengths were set at $240 \mathrm{~nm}$ at $0-15 \mathrm{~min}, 283 \mathrm{~nm}$ at $15-55 \mathrm{~min}$ and $270 \mathrm{~nm}$ at 25-35 min, and the absorption spectra of compounds were recorded from 190 to $400 \mathrm{~nm}$.

Preparation of JG-containing serum. A total of 20 male specific-pathogen-free Sprague-Dawley rats (age, 8-10 weeks; weight, $300 \pm 30 \mathrm{~g}$ ) were obtained from Shanghai Laboratory Animal Center (Shanghai, China) and provided with food and water ad libitum, at $20^{\circ} \mathrm{C}$ and $50 \%$ relative humidity, in a filtered clean atmosphere, under a12-h dark/light cycle. Rats were randomly divided into 2 groups ( $n=10 /$ group): i) JG group, which received a dose of JG ( $2 \mathrm{~g} / \mathrm{kg} / \mathrm{day})$ intragastrically twice per day for 8 days; and ii) Blank control group, which received a dose of standard saline $(10 \mathrm{ml} / \mathrm{kg} /$ day $)$ intragastrically twice per day for 8 days. Rats were anesthetized $1 \mathrm{~h}$ following the last gavage, with an intraperitoneal injection of $2 \%$ pentobarbital sodium $(0.2 \mathrm{ml} / 100 \mathrm{~g}$ body weight $)$ and euthanized by abdominal aorta exsanguination. The blood was collected and centrifuged at $3,000 \mathrm{xg}$ at room temperature for $15 \mathrm{~min}$ to obtain the serum. Serum samples were collected from each group, JG-containing serum or Blank serum, which were subsequently filtered through a $0.22-\mu \mathrm{m}$ filter membrane and stored in an ultralow freezer at $-80^{\circ} \mathrm{C}$. All experimental procedures were performed in accordance with the NIH
Guidelines for the Care and Use of Laboratory Animals and the National Animal Welfare Law of China. The present study was approved by the Ethics Committee of Fujian University of Traditional Chinese Medicine (Fuzhou, China).

Cell culture experiments. Osteoclast precursor RAW264.7 cells were obtained from The Cell Bank of Type Culture Collection of Chinese Academy of Science (Shanghai, China) and cultured in a differentiation medium comprising: $\alpha$-minimum essential medium ( $\alpha$-MEM) supplemented with $10 \%$ fetal bovine serum (FBS) (both from Gibco; Thermo Fisher Scientific, Inc., Waltham, MA, USA) and $50 \mathrm{ng} / \mathrm{ml}$ RANKL (R\&D Systems, Inc., Minneapolis, MN, USA). The culture medium was replaced every $48 \mathrm{~h}$. Cells were incubated at $37^{\circ} \mathrm{C}$ in a humid atmosphere containing $5 \% \mathrm{CO}_{2}$ for 6 days to obtain osteoclasts.

For differentiation assays, cells were seeded in 24-well plates at a density $1 \times 10^{4}$ cells/well in differentiation medium (without FBS) with various concentrations $(2,5,10,15$ and $20 \%$ ) of either JG-containing serum or Blank serum during the entire culture period of 6 days. The differentiation rate of each well was calculated and compared to determine the most effective concentration of JG-containing serum.

For RANK/RANKL pathway tests, cells were divided into 2 groups: i) The Blank group, which was treated with $50 \mathrm{ng} / \mathrm{ml}$ RANKL and 10\% Blank serum; and ii) the JG group, which was treated with $50 \mathrm{ng} / \mathrm{ml}$ RANKL and $10 \%$ JG-containing serum. Following 24, 48 and $96 \mathrm{~h}$ incubation, the cells were harvested for reverse transcription-quantitative polymerase chain reaction (RT-qPCR) and western blot analysis. Cells cultured in common medium ( $\alpha$-MEM supplemented with $10 \%$ Blank serum, without RANKL) as negative control ( $0 \mathrm{~h}$ of Blank group). All experiments were repeated at least 3 times.

Osteoclast differentiation assays. Osteoclast differentiation rate was measured by counting the number of tartrate-resistant acid phosphatase (TRAP)-positive stained cells, using the TRAP Staining kit (Nanjing Jiancheng Bioengineering Institute, Nanjing, China), according to the manufacturer's protocol. RAW264.7 cells cultured in differentiation medium at a density of $1 \times 10^{4}$ cells/well for 6 days were collected and fixed with the fixative solution in the kit at room temperature for $10 \mathrm{~min}$, rinsed thoroughly with deionized water and stained with naphthol AS-BI phosphate for $1 \mathrm{~h}$ at $37^{\circ} \mathrm{C}$, followed by hematoxylin counterstaining at room temperature for $2 \mathrm{~min}$. Osteoclasts were determined to be TRAP-positive stained multinuclear (containing $\geq 3$ nuclei) cells under light microscopy. A total of 6 fields/well (magnification, 100x) were examined.

To detect the F-actin containing podosome belt of osteoclasts, rhodaminephalloidin staining (1:200 dilution with PBS; Cytoskeleton Inc., Denver, CO, USA) was performed, according to the manufacturer's protocol. RAW264.7 cells cultured in a differentiation medium at a density of $1 \times 10^{4}$ cells/well for 6 days were collected, and fixed at room temperature with $4 \%$ paraformaldehyde for $10 \mathrm{~min}$, permeabilized with $1 \%$ Triton X-100 for 10 min, washed with PBS for $3 \mathrm{~min}$ and incubated with rhodaminephalloidin at room temperature in the dark for $30 \mathrm{~min}$, followed by 3 washes with PBS for 5 min each. Nuclei were counterstained with $100 \mathrm{nM}$ DAPI in PBS at room temperature for 5 min. Osteoclasts were 

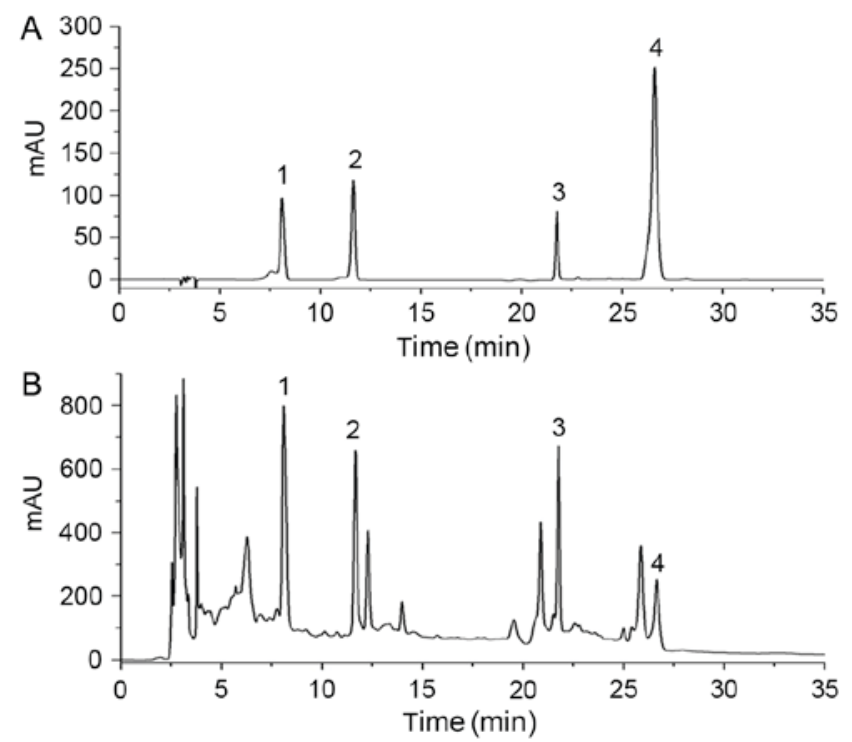

Figure 1. High-performance liquid chromatographychromatogram of JG. (A) Mixed standard solution of four components of JG. (B) JG sample analysis. 1, morroniside; 2, loganin; 3, aurantiamarin; 4, icariin; JG, jiangu granule; mAU, milli-absorbance units.

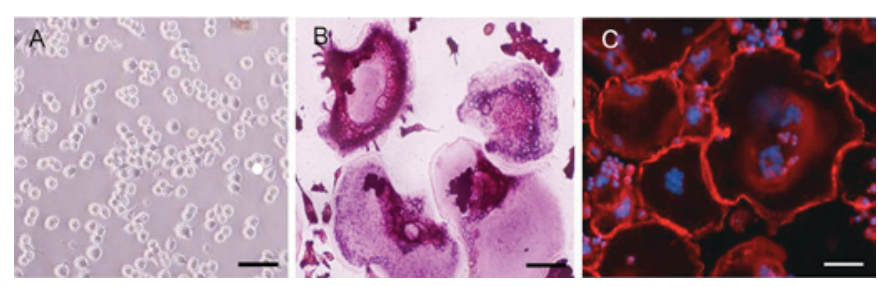

Figure 2. Osteoclast differentiation in the presence of RANKL. (A) Untreated RAW264.7 cells. (B) Tartrate-resistant acid phosphatase staining of RAW264.7 cells treated with RANKL (50 ng/ml) for 6 days. (C) F-actin staining by rhodaminephalloidin (red) of RAW264.7 cultures treated with RANKL ( $50 \mathrm{ng} / \mathrm{ml}$ ) for 6 days; nuclei were counterstained with DAPI (blue) Scale bar, $50 \mu \mathrm{m}$. RANKL, receptor activator of nuclear factor- $\kappa \mathrm{B}$ ligand.

observed under a fluorescence microscope (Leica DMI4000B; Leica Microsystems GmbH, Wetzlar, Germany).

$R T$-qPCR for RANK/RANKL pathway components. Cells from the Blank control group, JG group and negative control group were collected. RNA was extracted by TRIzol (Invitrogen; Thermo Fisher Scientific, Inc.) from cells at a density of $1 \times 10^{7}$ cells $/ \mathrm{ml}$ and reverse transcribed to cDNA using Prime Script First-Strand cDNA Synthesis kit (Takara Biotechnology Co., Ltd., Dalian, China) for use as qPCR template. The primers were as follows: RANK forward, 5'-GGCTGGCTACCACTGGAACT-3' and reverse, 5'-TCC TGTAGTAAACGCCGAAGA-3'; TRAF6 forward, 5'-TCA TTATGATCTGGACTGCCCAAC-3' and reverse, 5'-TTA TGAACAGCCTGGGCCAAC-3'; NF- $\kappa$ B forward, 5'-ACC ACTGCTCAGGTCCACTGTC-3' and reverse, 5'-GCTGTC ACTATCCCGGAGTTCA3-'; NFATc1, 5'-CAAGTCTCA CCACAGGGCTCACTA-3' and reverse, 5'-TCAGCCGTC CCAATGAACAG-3';c-Fos forward, 5'-ACGTGGAGCTGA AGGCAGAAC-3' and reverse, 5'-AGCCACTGGGCCTAG ATGATG-3'; and $\beta$-actin forward, 5'-AGGCTGTGTTGT CCCTGTA-3' and reverse, 5'-ATGTCACGCACGATTTCC

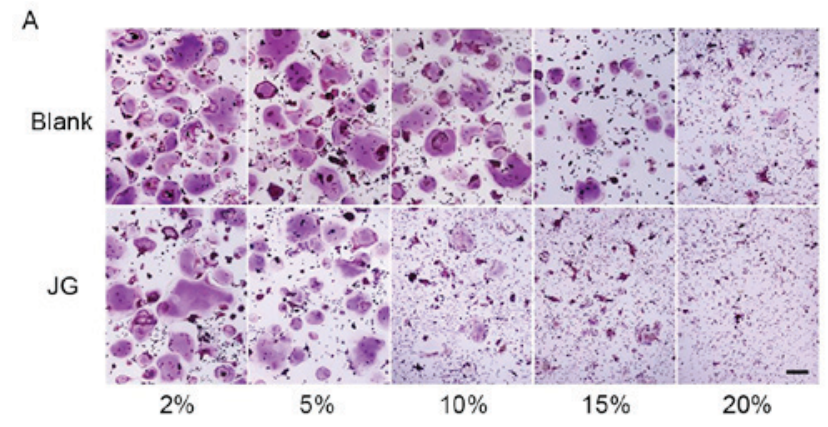

B

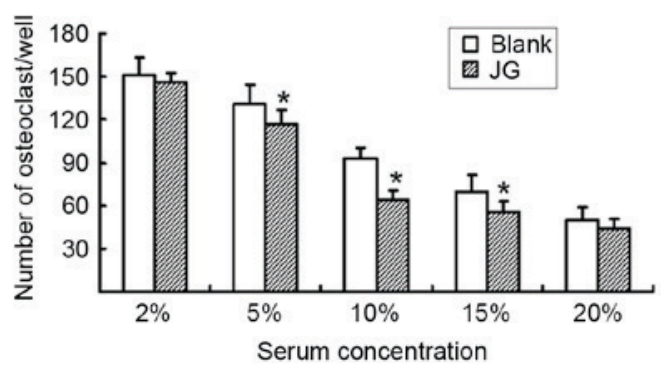

Figure 3. Osteoclast formation of RAW264.7 treated with various concentrations of JG-containing serum in the presence of RANKL. (A) RAW264.7 cells were treated with RANKL $(50 \mathrm{ng} / \mathrm{ml})$ for 6 days, and examined using TRAP staining. (B) Total number of TRAP-positive stained cells, which indicates differentiated osteoclasts. Data are presented as the mean \pm standard deviation of 3 cultures. ${ }^{*} \mathrm{P}<0.05$ vs. Blank-serum group. TRAP, tartrate-resistant acid phosphatase.

-3'. PCR was performed using SYBR Green qPCR Mix (Takara Biotechnology Co., Ltd.) and a Real-Time PCR system (ABI7500; Thermo Fisher Scientific, Inc.) with the following program: 1 cycle at $95^{\circ} \mathrm{C}$ for $30 \mathrm{sec}$, followed by 40 cycles of $95^{\circ} \mathrm{C}$ for $5 \mathrm{sec}, 60^{\circ} \mathrm{C}$ for $34 \mathrm{sec}$. qPCR was carried out on three replicates per sample. $\beta$-actin was used as a reference gene for RNA correction of all samples; the relative standard curve method ( $2^{-\Delta \Delta \mathrm{Cq}}$ method) was used for the calculation of fold changes in gene expression (12).

Western blot analysis for RANK/RANKL pathway. Cells from the Blank control, JG and negative control groups were collected. Total protein was extracted in protein lysis buffer (Beyotime Institute of Biotechnology, Haimen, China) from cells at a density of $5 \times 10^{7}$ cells $/ \mathrm{ml}$. Protein concentrations were determined by BCA Protein assay kit (Beyotime Institute of Biotechnology). Equal amounts of proteins $(30 \mu \mathrm{g})$ were resolved by SDS-PAGE on a $12 \%$ gel and transferred to polyvinylidene difluoride membranes (EMD Millipore, Billerica, MA, USA). Non-specific interactions were blocked with 5\% skim milk at $4^{\circ} \mathrm{C}$ for $2 \mathrm{~h}$ and the membranes were incubated with 1:500 diluted primary antibodies [rabbit anti-RANK polyclonal antibody (cat no. ab200369), rabbit anti-TRAF6 monoclonal antibody (cat no. ab33915), rabbit anti-NF- $\mathrm{kB}$ p105/p50 monoclonal antibody (cat no. ab32360), rabbit anti-NFATc1 polyclonal antibody (cat no. ab25916), rabbit anti-c-Fos polyclonal antibody (cat no. ab190289), Rabbit anti- $\beta$-Actin monoclonal antibody (cat. no. ab8227) all Abcam, Cambridge, MA, USA]. Rabbit anti-NF-kB p100/p52 monoclonal antibody (cat no. 52583; Cell Signaling Technology, Inc., MA, USA) overnight at $4^{\circ} \mathrm{C}$, followed by incubation with horseradish peroxidase (HRP)-conjugated secondary 

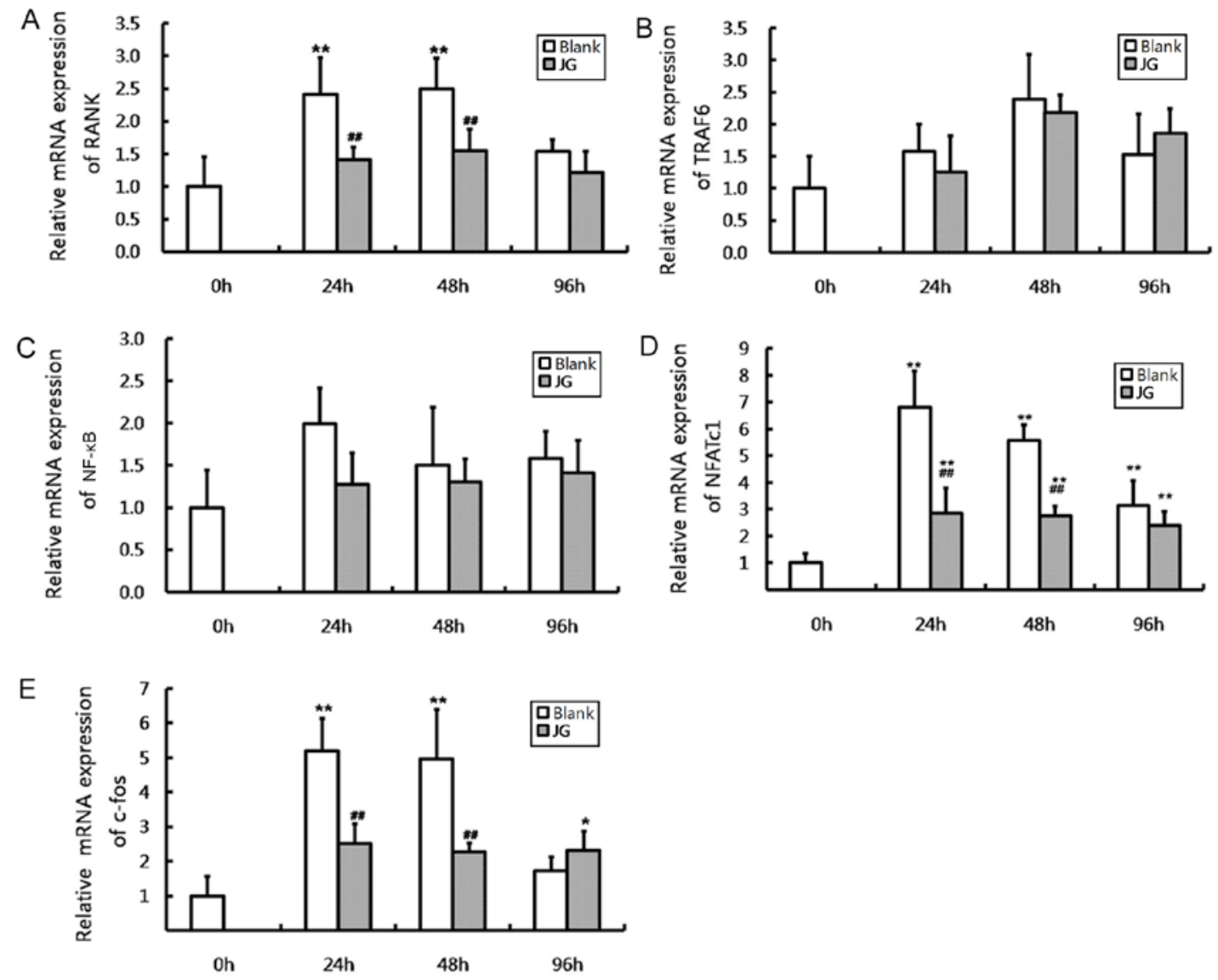

Figure 4. Effects of JG-containing serum on mRNA expression of RANK/RANKL pathway components in RAW264.7 cells. Results of reverse transcription-quantitative polymerase chain reaction indicating the relative mRNA expression levels of (A) RANK, (B) TRAF6, (C) NF- $\kappa$ B, (D) NFATc1 and (E) c-Fos. Data are presented as the mean \pm standard deviation of 3 cultures. ${ }^{*} \mathrm{P}<0.05$ vs. Blank group, ${ }^{* *} \mathrm{P}<0.01$ vs. Blank group; ${ }^{*} \mathrm{P}<0.05$ vs. negative control $(0 \mathrm{~h}$ of Blank group), ${ }^{\#} \mathrm{P}<0.01$ vs. negative control (0 h of Blank group). JG, jiangu granule; NFATc1, nuclear factor of activated T cells, cytoplasmic 1; NF- $\kappa \mathrm{B}$, nuclear factor- $\kappa \mathrm{B}$; RANK, receptor activator of nuclear factor- $\kappa \mathrm{B}$ ligand; RANKL, RANK ligand; TRAF6, tumor necrosis factor receptor-associated factor 6.

antibodies (goat anti-rabbit immunoglobulin G H\&L HRP; 1:5,000; cat no. ab6721; Abcam, Cambridge, MA, USA). Protein bands were visualized with the enhanced chemiluminescence reagent (Invitrogen; Thermo Fisher Scientific, Inc.). Densitometric values were quantified for each band with the Image Pro-Plus program (version 5.0; Media Cybernetics, Inc., Rockville, MD, USA). Relative expression data are expressed as a ratio of the optical intensity of the band of the target protein over that of the internal control protein ( $\beta$-actin).

Statistical analysis. All calculations were performed using SPSS version 17.0 for Windows software (SPSS Inc., Chicago, IL, USA). Results are presented as the mean \pm standard deviation. All data were analyzed using one-way analysis of variance and Fisher's least significant difference test. $\mathrm{P}<0.05$ were considered to indicate a statistically significant difference.

\section{Results}

HPLC analysis of JG. For HPLC analysis, four standard compounds (Fig. 1A) were used to determine the composition of the JG extract (Fig. 1B), including morroniside and loganin from C.officinalis Sieb. et Zucc., aurantiamarin from Pericarpium Citri Reticulatae and icariin from E. brevicornum Maxim.
RANKL induces osteoclastogenesis. The results of TRAP staining demonstrated a notable increase in TRAP-positive multinuclear cells in the RAW264.7 cells incubated with RANKL for 6 days (Fig. 2A and B). Immunofluorescence staining with rhodaminephalloidin revealed multinucleated giant cells with the characteristic podosome belt of osteoclasts (Fig. 2B and C). These results indicated that RANKL treatment induced the formation of osteoclasts from RAW264.7 cells.

$J G$-containing serum inhibits osteoclast differentiation induced by RANKL. The differentiated osteoclasts were treated with various concentrations of either JG-containing serum or Blank serum for 6 days, and subsequently stained with TRAP (Fig. 3A). The number of TRAP-positive stained cells in the 5, 10 and $15 \%$ JG-containing serum groups were significantly decreased compared with the number of TRAP-positive cells in the Blank group $(\mathrm{P}<0.05$; Fig. $3 \mathrm{~B})$ particularly at the concentration of $10 \%$, which demonstrated that the serum dose-dependently decreased the differentiation rate of osteoclasts.

Effects of JG-containing serum on RANK/RANKL pathway. The results of RT-qPCR and western blot analysis were consistent compared with the negative control $(0 \mathrm{~h}$ of Blank 

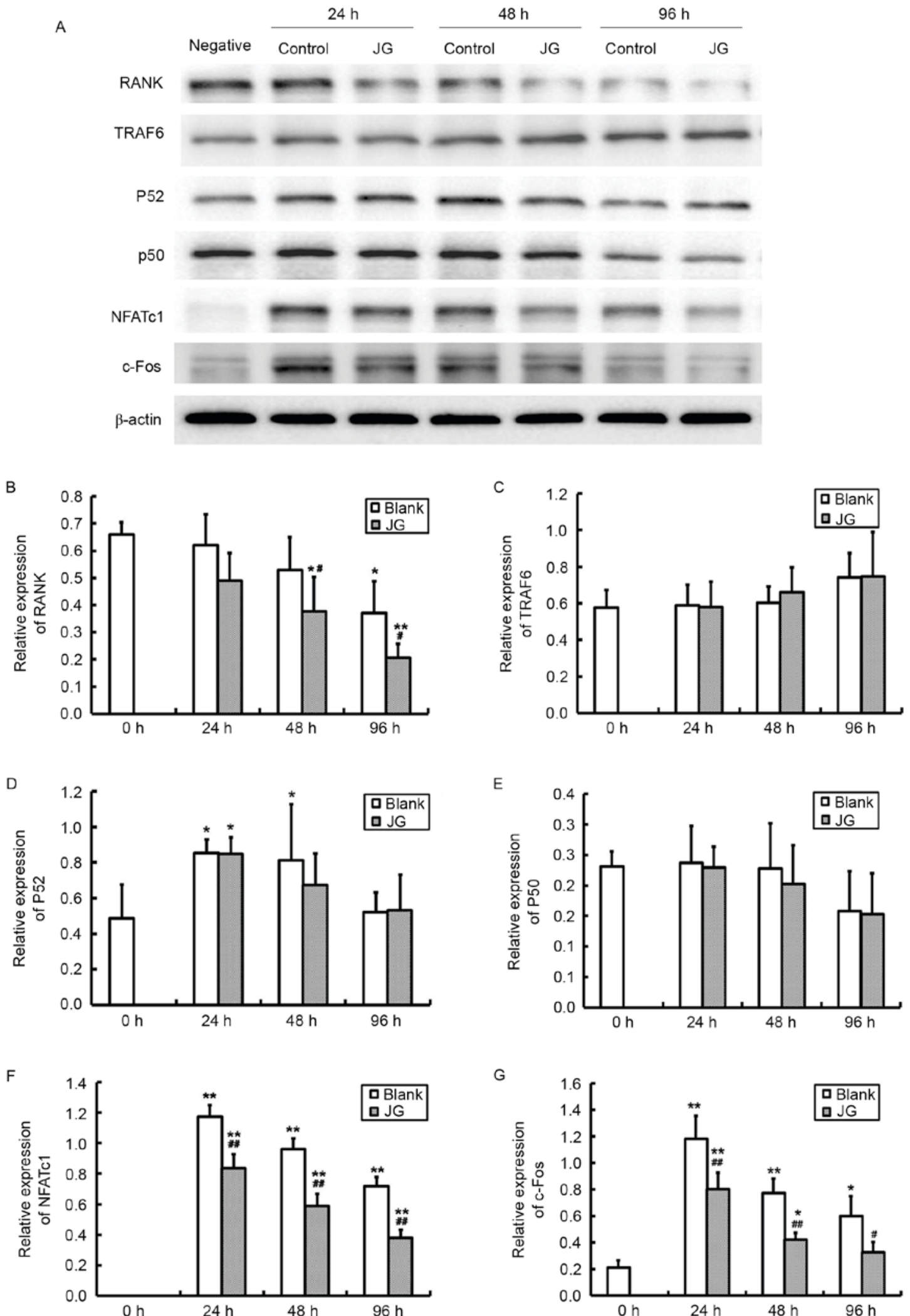

Figure 5. Effects of JG-containing serum on protein expression levels of RANK/RANKL pathway components. (A) Images of the protein expression as analyzed by western blot assay. Relative protein expression levels of (B) RANK, (C) TRAF6, (D) NF-kBp52, (E) NF-kBp50, (F) NFATc1 and (G) c-Fos. Data are presented as the mean \pm standard deviation of 3 cultures. ${ }^{*} \mathrm{P}<0.05$ vs. Blank group; ${ }^{* * *} \mathrm{P}<0.01$ vs. Blank group; ${ }^{*} \mathrm{P}<0.05$ vs. negative control $(0 \mathrm{~h}$ of $\mathrm{Blank}$ group); ${ }^{\# \#} \mathrm{P}<0.01$ vs. negative control ( $0 \mathrm{~h}$ of Blank group). JG, jiangu granule; NFATc1, nuclear factor of activated T cells, cytoplasmic 1; NF-kB, nuclear

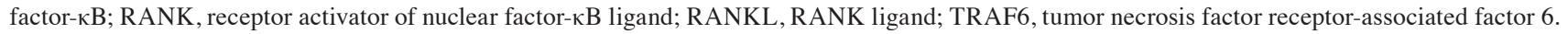

group), the mRNA and protein expression levels of NF- $\mathrm{kB}$, NFATc1 and c-Fos significantly increased in JG-containing serum group and the Blank serum group following 24, 48 and $96 \mathrm{~h}$ of RANKL stimulation $(\mathrm{P}<0.01)$. Compared with Blank 
group, the mRNA and protein expression of RANK, NFATc1 and c-Fos of JG group significantly decreased following treatment for 24 and $48 \mathrm{~h}(\mathrm{P}<0.01)$. The differences ofTRAF6 and $\mathrm{NF}-\kappa \mathrm{B}$ expression between the Blank group and JG group were not significant (Figs. 4 and 5).

\section{Discussion}

In TCM it is believed that the kidney is the origin of congenital constitution. Bones are governed and nourished by the kidney, which means that the development and the quality of bones depend on the functions of the kidneys (13). It is been reported that several traditional Chinese kidney-tonifying herbals may have bone-strengthening effects (14). It is also believed in TCM that the spleen is the origin of the acquired constitution, which nourishes the kidney. The kidney and spleen complement each other, so as to maintain bone health. A summary of ancient literatures on constitution during menopause has indicated that a shared pathogenesis in PMOP may be kidney deficiency, and is frequently accompanied with spleen deficiency; hence, the basic law of bone strengthening is to reinforce them $(8,9)$. Based on its pharmacological effects of tonifying kidney and spleen, the Chinese formula JG was applied to prevent and treat PMOP.

Bone is a dynamic organ that undergoes continuous remodeling; that is, the osteoclasts resorb old and damaged bone, which is replaced with new bone by osteoblasts $(15,16)$. The activity and balance of osteoclasts and osteoblasts maintain the structural integrity and bone mass. When bone resorption surpasses formation, bone mass decreases and osteoporosis may occur. PMOP is a disease that results from dramatic bone loss and increased bone destruction, owing to enhanced osteoclastogenesis without a corresponding increase in osteoblastic activity (2-4). The mouse monocyte/macrophage cell line RAW264.7, widely used as osteoclast precursor, exhibits a strong potential to differentiate into osteoclasts in the presence of RANKL (17). TRAP staining and F-actin immunofluorescence staining are usually used for osteoclast identification due to the abundant TRAP expression and podosome belt formation of mature osteoclasts (18). Results from the present study indicated that JG-containing serum treatment reduced the RANKL-induced osteoclast differentiation from RAW264.7, which indicated that JG may prevent osteoporosis by inhibiting osteoclastogenesis and osteoclastic bone resorption.

Osteoclasts are large, multinucleated cells that are derived from the monocyte/macrophage lineage. The RANK/RANKL pathway serves a crucial role in osteoclast differentiation and bone resorption (6,7). RANKL induces intracellular signals through its receptor, RANK, and regulates the expression of various down stream signaling molecules to exert their osteoclast-inducing effect $(19,20)$. The cytoplasmic domain of RANK binds TRAF1, TRAF2, TRAF3, TRAF5, and TRAF6 to mediate the signals (21). TRAF6 is the most important of the TRAFs (22), which transmits signals to downstream targets such as $\mathrm{NF}-\kappa \mathrm{B}$ and the mitogen-activated protein kinase (MAPK) signaling pathways $(23)$. $N F-\kappa B$ regulates target genes with its binding sites through classical or non-classical pathway activation. There are five members of the $N F-\kappa B$ family in mammals: p50/p105, p65/RelA, c-Rel, RelB and p52/p100. p50 and p52 expression is required for osteoclast precursors to differentiate into osteoclasts in response to RANKL (24,25). MAPK signaling pathways activate transcription factors of the activator protein 1 family, which includes Jun, Fos and Fos-related antigen, which subsequently regulate the expression of necessary and important genes for osteoclast differentiation. c-Fos is activated by a number of growth factors and cytokines, and serves a key role in RANKL-induced osteoclast differentiation. c-Fos gene deficient mice exhibited serious bone sclerosis owing to a complete block of osteoclast differentiation (26). In addition, c-Fos induces and activates NFATc1, which is another key transcription factor that affects osteoclast differentiation (27). NFATc1 is activated by calcium signaling and binds to its own promoter, thus turning on an autoregulatory loop. NFATc1 also promotes expression and activation of osteoclast-specific genes and proteins like TRAP, calcitonin receptor and cathepsin, which results in the termination of osteoclastic differentiation.

In the present study, key molecules of the RANK signaling pathway, including RANK, TRAF6, NF- $\mathrm{B}$ (p50 and p52), NFATc1 and c-Fos were examined to investigate the effects of JG treatment on RANKL-induced osteoclastogenesis. The results demonstrated that RANKL stimulation increased mRNA and protein expression of NF- $\kappa$ B, c-Fos and NFATc1, and consequently led to osteoclast differentiation, which was consistent with the results of previous studies $(19,20)$. It was also revealed that JG-containing serum significantly reduced the expression of RANK, c-Fos and NFATc1, and consequently inhibited RANKL-induced osteoclastogenesis. Decreased osteoclastogenesis reduces bone resorption and ultimately increases bone mass. Therefore, the results indicated a possible mechanism of JG on preventing and treating PMOP.

In conclusion, the present study demonstrated the effects of JG treatment on inhibiting osteoclast differentiation, which may be achieved through the RANK/RANKL signaling pathway. This study provides an experimental rationale for the application of JG in clinical therapy of PMOP.

\section{Acknowledgements}

This work was supported by the Natural Science Foundation of China (grant nos. 81574003 and 81473706), the Guiding Project Foundation of Fujian Science and Technology Department (grant no. 2015Y0069) and the Science Foundation of Fujian Province (grant nos. 2015J01690 and 2014J01355).

\section{References}

1. Consensus development conference: Diagnosis, prophylaxis, and treatment of osteoporosis. Am J Med 94: 646-650, 1993.

2. Pacifici R: Estrogen, cytokines, and pathogenesis of postmenopausal osteoporosis. J Bone Miner Res 11: 1043-1051, 1996.

3. Seifert-Klauss V, Fillenberg S, Schneider H, Luppa P, Mueller D and Kiechle M: Bone loss in premenopausal, perimenopausal and postmenopausal women: Results of a prospective observational study over 9 years. Climacteric 15: 433-440, 2012.

4. de Villiers TJ: Bone health and osteoporosis in postmenopausal women. Best Pract Res Clin Obstet Gynaecol 23: 73-85, 2009.

5. Tella SH and Gallagher JC: Prevention and treatment of postmenopausal osteoporosis. J Steroid Biochem Mol Biol 142: 155-170, 2014.

6. Lacey DL, Boyle WJ, Simonet WS, Kostenuik PJ, Dougall WC, Sullivan JK, San Martin J and Dansey R: Bench to bedside: Elucidation of the OPG-RANK-RANKL pathway and the development of denosumab. Nat Rev Drug Discov 11: 401-419, 2012. 
7. Bridgeman MB and Pathak R: Denosumab for the reduction of bone loss in postmenopausal osteoporosis: A review. Clin Ther 33: 1547-1559, 2011.

8. Wang XX, Zhang YL and Huang QF: Discussion on the main pathogenesis in traditional Chinese medicine and etiology about primary osteoporosis. Zhong Xi Yi Jie He Xue Bao 8: 1119-1123, 2010 (In Chinese)

9. Li DT, Li FY, Wang J, Liu JH, Yan N, Cheng YM, Hu AH, Jiang HY, Shi FL, Zhang MZ, et al: A study of diagnostic criteria for traditional Chinese medicine syndromes in osteoporosis Zhong Xi Yi Jie He Xue Bao 9: 1326-1332, 2011 (In Chinese).

10. Lin YP, Zhou RX and Guo SM: Effect of jiangu granule on quality of bone in model rats with osteoporosis induced by ovariectomy. Zhongguo Zhong Xi Yi Jie He Za Zhi 24: 431-434, 2004 (In Chinese).

11. Lin YP, Ma JH and Feng EY: Study on preventive effect of jiangu granule on osteoporosis in ovariectomized rats. Zhongguo Zhong Xi Yi Jie He Za Zhi 22: 369-371, 2002 (In Chinese).

12. Livak KJ and Schmittgen TD: Analysis of relative gene expression data using real-time quantitative PCR and the 2(-Delta Delta C(T)) method. Methods 25: 402-408, 2001.

13. Ju D, Liu M, Zhao H and Wang J: Mechanisms of 'kidney governing bones' theory in traditional Chinese medicine. Front Med 8: 389-393, 2014

14. Li Y, Tong J, Zhou YJ and Xu XY: Research progress on anti-osteoporotic active ingredients and pharmacological action mechanism of traditional Chinese kidney-tonifying and bone-strengthening drugs. Zhongguo Zhong Yao Za Zhi 40: 1038-1043, 2015 (In Chinese).

15. Crockett JC, Rogers MJ, Coxon FP, Hocking LJ and Helfrich MH: Bone remodelling at a glance. J Cell Sci 124: 991-998, 2011.

16. Hadjidakis DJ and Androulakis II: Bone remodeling. Ann NY Acad Sci 1092: 385-396, 2006

17. Kartsogiannis $\mathrm{V}$ and $\mathrm{Ng} \mathrm{KW}$ : Cell lines and primary cell cultures in the study of bone cell biology. Mol Cell Endocrinol 228: 79-102, 2004.
18. Väänänen HK, Zhao H, Mulari M and Halleen JM: The cell biology of osteoclast function. J Cell Sci 113: 377-381, 2000.

19. Liu C, Walter TS, Huang P, Zhang S, Zhu X, Wu Y, Wedderburn LR, Tang P, Owens RJ, Stuart DI, et al: Structural and functional insights of RANKL-RANK interaction and signaling. J Immunol 184: 6910-6919, 2010.

20. Kuroda $Y$ and Matsuo K: Molecular mechanisms of triggering, amplifying and targeting RANK signaling in osteoclasts. World J Orthop 3: 167-174, 2012.

21. Inoue Ji, Ishida T, Tsukamoto N, Kobayashi N, Naito A, Azuma S and Yamamoto T: Tumor necrosis factor receptor-associated factor (TRAF) family: Adapter proteins that mediate cytokine signaling. Exp Cell Res 254: 14-24, 2000

22. Armstrong AP, Tometsko ME, Glaccum M, Sutherland CL, Cosman D and Dougall WC: A RANK/TRAF6-dependent signal transduction pathway is essential for osteoclast cytoskeletal organization and resorptive function. J Biol Chem 277: 44347-44356, 2002.

23. Asagiri $\mathrm{M}$ and Takayanagi $\mathrm{H}$ : The molecular understanding of osteoclast differentiation. Bone 40: 251-264, 2007

24. Xing L, Bushnell TP, Carlson L, Tai Z, Tondravi M, Siebenlist U, Young F and Boyce BF: NF-kappaB p50 and p52 expression is not required for RANK-expressing osteoclast progenitor formation but is essential for RANK- and cytokine-mediated osteoclastogenesis. J Bone Miner Res 17: 1200-1210, 2002.

25. Soysa NS and Alles N: NF-kappaB functions in osteoclasts. Biochem Biophys Res Commun 378: 1-5, 2009.

26. Matsuo K, Owens JM, Tonko M, Elliott C, Chambers TJ and Wagner EF: Fosl1 is a transcriptional target of c-Fos during osteoclast differentiation. Nat Genet 24: 184-187, 2000.

27. Zhao Q, Wang X, Liu Y, He A and Jia R: NFATc1: Functions in osteoclasts. Int J Biochem Cell Biol 42: 576-579, 2010. 